\title{
Fibromialgia y/o Síndrome de la Fatiga Crónica. Una propuesta para la aproximación a su doble dimensión jurídica y médica ${ }^{1}$
}

\author{
Javier León Iglesias \\ Abogado \\ Licenciado en Derecho por la Universidad de Deusto \\ Doctor en Medicina por la Universidad del País Vasco
}

\begin{abstract}
Resumen: En los últimos años hemos venido presenciando grandes cambios en relación con las enfermedades crónicas, tanto cuantitativa -incremento significativo de afectados - , como cualitativamente - distintas formas de enfermar-. Surgen así las realidades clínicas derivadas de la Fibromialgia - FM- y/o Síndrome de la Fatiga Crónica - SFC-.

Con unas particularidades clínicas similares —dolor músculo-esquelético difuso crónico y fatiga severa-, cuentan con una elevada epidemiología y una dificultad para su objetivación, pues las pruebas diagnósticas suelen arrojar resultados de práctica normalidad, lo cual engendra serios problemas para su consideración como entidades socio-laborales, conforme a la definición legal de Incapacidad Permanente.
\end{abstract}

Previo estudio de 77 expedientes de invalidez en los que figuraban estos diagnósticos, nos encontramos con personas que desempeñaban, en su mayoría, profesiones de contenido físico, y que padecían un mayor número de entidades artrósicas, herniarias y ansioso-depresivas respecto al colectivo general de sujetos con estos procesos patológicos.

Resulta aún muy discutida, en el ámbito clínico y jurídico, la influencia que tienen en la FM y/o SFC las alteraciones psicológicas, no así su importante coste farmacológico - la polimedicación observada representa 1.634,19 euros por persona y año- - y prestacional —un $14,66 \%$ del conjunto de pensiones de Incapacidad Permanente en los grados de Absoluta y Total que abona el INSS-.

Palabras clave: Fibromialgia, fatiga crónica, incapacidad laboral.

Abstract: During recent years we have been able to observe significant changes in the field of chronic diseases, both in form (different ways to acquire the illness) and factor (significant increase in the number of affected subjects). As such, new clinical realities taking after Fibromyalgia -FM- and Chronic Fatigue Syndrome - CFS—, are happening.

${ }^{1}$ A los Profesores Ricardo Franco y Fernando Manrique. El primero de ellos me acercó al apasionante mundo de la ciencia médica, mientras que debo al segundo mi formación como jurista. Ambos me enseñaron que la persona que desempeña cualquier tipo de modalidad profesional es precisamente, y antes que cualquier otra cosa, eso: persona. 
With similar clinical specificities - chronic diffuse muscle-skeleton pain, and severe fatigue-, that account for a high epidemiologic base, and pose serious difficulties as to their objectification, since diagnosis tests will normally throw up results of practical normality, it represents a serious problem as to their being considered socio-labour entities, in accordance with the legal definition of Permanent Disability.

Following a study of 77 disability dossiers, where these diagnosis were to be found, we encountered subjects who were in the majority of cases engaged in physical related professions, and who were subject to a higher number or arthrosis, hernia, and anxious-depressive states than those affecting the broader population with similar patogenous processes.

It is still highly debated within the legal and medical arena, the influence FM and CFS may have in a psychological disorder, even though its high pharmacological cost is a proven fact -observed polymedication represents 1.634,19 euros per head/year - as it also is benefit wise - accounting for up to 14,66\% of the global figure of Absolute and Total Permanent Disability Pensions, being handed over by the INSS-.

Key words: Fibromyalgia, Chronic Fatigue, Work disability.

En los albores del siglo XXI, el Derecho tiene la misión de dar respuesta, ahora más que nunca, a una nueva, a la vez de compleja, dinámica social, uno de cuyos principales paradigmas - un logro de la Medicina- es que contamos con una esperanza de vida más larga; si bien la otra cara de la moneda la encontramos en el panorama epidemiológico actual sobre enfermos crónicos y su correlato necesario en el ámbito de las incapacidades laborales. No en vano, los datos de la encuesta sobre discapacidades, deficiencias y estado de Salud (EDDES) recogidos por el Instituto Nacional de Estadística (INE) ${ }^{1}$, revelan que en torno a 19 millones de españoles padecen procesos patológicos crónicos, de los cuales tan sólo el 20,7\% permanecen en activo laboral.

Los cambios sociales que estamos presenciando en los últimos años afectan, incluso, a las formas de enfermar. Sirva como ejemplo ${ }^{2}$ la epidemia ocurrida a mediados de la década de los ochenta en el sector de operadores de teléfono y teclados - Telecom Australia — en la que, sin motivo aparente, un buen número de personas presentaban una serie de menoscabos dolorosos, ocasionados - se pensaba entonces - por esfuerzos repetitivos, lo que motivó múltiples reclamaciones indemnizatorias, procedimientos judiciales en definitiva, por esta pérdida de la capacidad laboral.

Hoy sabemos que este acontecimiento, que trajo de cabeza a los operadores médico-legales de aquel país, pudo atribuirse a las entidades clínicas que ahora nos ocupan: la Fibromialgia —en adelante FM- y/o Síndrome de la Fatiga Crónica - SFC-

Fue Hench quien en 1975 incorporó el vocablo FM, si bien habríamos de esperar hasta 1990 para que el American College of Rheumatology ${ }^{3}$ fijara los 
criterios para su clasificación, cuyo eje fundamental lo representan los 18 puntos - tender points - distribuidos en la anatomía corporal del sujeto evaluado - se reproducen en la Tabla número $1-$, de los cuales al menos 11 deben ser sumamente dolorosos a la palpación por el facultativo. En consecuencia, podemos definir la $\mathrm{FM}^{4,5}$ como un trastorno caracterizado por la aparición de dolor músculo-esquelético difuso crónico que, a menudo, se acompaña de un cortejo sintomático de, entre otros, rigidez matutina, sueño no reparador, fatiga y debilidad, cefaleas, colon irritable, fenómeno de Raynaud, síndrome seco y trastornos emocionales. Aunque sus mecanismos de producción son hoy por hoy desconocidos, el mayor consenso doctrinal ${ }^{6}$ viene a considerar que lo que en realidad ocurre es que se pone de manifiesto una disfunción en los mecanismos de la modulación y de la percepción del dolor en proporciones superiores a lo que se considera como clínicamente normal, habiéndose comprobado, a su vez, un trastorno neurológico caracterizado por el incremento de los tonos simpáticos y descenso de los parasimpáticos ${ }^{7}$.

Desde su reconocimiento por la Organización Mundial de la Salud, OMS, en 1992, y su consiguiente tipificación en el manual de clasificación internacional de enfermedades (ICD 10) con el código M79-0, su prevalencia ha ido aumentando progresivamente. En nuestro país, el trabajo publicado por la Sociedad Española de Reumatología ${ }^{8}$ habla en favor de un nivel aproximado de afectación del 2,37\% en personas mayores de 20 años — con una relación mujer-varón de 21-1—, por lo que se estima que alrededor de 700.000 españoles cumplen con los criterios de la FM.

Una clínica muy similar a la del SFC — por lo que en este trabajo abordamos conjuntamente ambas realidades clínicas-, cuyos criterios - esta vez diagnósticos—- se fijan en $1994^{9}$ — Tabla número 2 - . Nos encontramos, pues, ante una entidad clínica a la que algunos trabajos ${ }^{10}$ atribuyen una epidemiología de un 2,6\% de la población general, y que se caracteriza por fatiga severa incapacitante en combinación con otros síntomas: trastornos en la concentración y en la memoria breve, alteraciones del sueño y dolor músculo-esquelético.

Sentados estos presupuestos, el lector posiblemente se preguntará qué papel juega el Derecho y, más concretamente, el Derecho de la Seguridad Social como disciplina autónoma ${ }^{11}$, en el ámbito de estas patologías. La respuesta — descrita en la literatura médica cuando se apunta a la, también, alta incidencia en el ámbito laboral, habida cuenta de que los sujetos afectados forman parte, en su mayoría, de la población activa ${ }^{12,13}$ — viene de la mano de la propia práctica jurídica, cuando los operadores intervinientes en este tipo de procesos se cuestionan la conveniencia o no de las incapacidades laborales.

Y centrado en estos términos el debate, proponemos un sistema de investigación en consonancia con una de las principales funciones del Derecho, que es la de ajustarse a las demandas sociales de cada momento. Parece cla- 
ro, en este sentido, que, si atendiéramos exclusivamente a los posicionamientos jurisprudenciales sobre la materia, estaríamos obviando no solamente los resultados de las investigaciones clínicas - con el quantum estadístico obtenido sobre los datos que se infieren de la entrevista clínica, exploración física o la terapéutica-, sino que también la opinión, igualmente cualificada, que tienen los facultativos sobre el eventual reconocimiento de la prestación de Incapacidad Permanente a personas afectadas de FM y/o SFC.

El punto de partida necesario de esta dinámica de trabajo debe ser, por ende, el análisis del panorama normativo y jurisprudencial de la pensión de referencia en nuestro país. Así las cosas, conforme al artículo 136.1 del Real Decreto Legislativo 1/1994 de 20 de junio, por el que se aprueba el Texto Refundido de la Ley General de la Seguridad Social _-LGSS_, en la modalidad contributiva, es Incapacidad permanente la situación del trabajador que, después de haber estado sometido al tratamiento prescrito y de haber sido dado de alta médicamente, presenta reducciones anatómicas o funcionales graves, susceptibles de determinación objetiva y previsiblemente definitivas, que disminuyan o anulen su capacidad laboral (...). El primer pasaje de la definición legal que genera controversia en el ámbito de los síndromes que nos ocupan es el que apunta a que las lesiones han de ser determinadas objetivamente, pues un nuevo elemento de identificación entre la FM y el SFC es el que trae causa en la práctica normalidad de las pruebas analíticas y complementarias de diagnóstico, que es menester realizar para acreditar que los síntomas existen y son tan severos como la persona afectada apunta en la anamnesis —entrevista clínica- No se ponen de acuerdo las publicaciones jurídicas de los últimos años sobre el verdadero alcance que debe conferirse a esta determinación objetiva, pues, aun cuando algunos ${ }^{14}$ informan de que para comprobar las reducciones anatómicas o funcionales deben practicarse pruebas médicas fiables —resonancia magnética, radiografías...- otros, por el contrario ${ }^{15}$, utilizan la disyuntiva determinación objetiva o diagnóstico del proceso patológico presente y comprobado, es decir, podría encajar en la acción protectora del precepto la confirmación médica del padecimiento, aunque las pruebas clínicas no arrojen datos patológicos —en este sentido, también, las sentencias del Juzgado de lo Social n. ${ }^{\text {o }} 32$ de Barcelona de 4-2-02, y del Juzgado de lo Social n. ${ }^{\circ} 3$ de Álava de 8-10-02-.

Nuestra Sala de lo Social del Tribunal Superior de Justicia del País Vasco acoge esta última doctrina —-sentencia de 18 de marzo de 2003 - añadiendo que las alteraciones sensoriales — de las que el dolor es la más habitual- son deficiencias a tener en cuenta, según lo dispuesto en el artículo 136.2 de la LGSS. Ahora bien, dudamos de la aplicación del precepto invocado, habida cuenta de que define el alcance prestacional de la Invalidez, pero en su modalidad no contributiva. De cualquier forma, este órgano judicial no siempre ha mantenido el mismo criterio. Recordemos, en este sentido, aquellas sentencias que informaban de que en la FM no constaba patolo- 
gía orgánica, concreta y determinada que la justificara, por lo que no encajaba dentro de los supuestos objetivables a que se refiere el artículo 136.1 de la LGSS —resoluciones judiciales de 11-2-03, recurso n. $.^{\circ} 23 / 03$, y de 29-4-03, Recurso n. ${ }^{\circ}$ 691/03, entre otras-.

Por su parte, no siempre es precisa el alta médica para acceder a la Incapacidad Permanente, según la literalidad del artículo - tras haber permanecido el beneficiario en Incapacidad Temporal durante un período de 12 meses, prorrogables por 6 más, y, llegando, en supuestos de demora de la calificación de Invalidez, hasta un máximo de 30 , todo ello según lo dispuesto en los artículos 128 y 131 Bis $2 .^{\circ}$, párrafo $2 .^{\circ}$ de la LGSS-, por cuanto que además de la situación de no alta restringida al acceso a la Incapacidad Permanente en el grado de Absoluta - de la que luego trataremos- o Gran Invalidez, conforme se dispone en el artículo 138.3 de la LGSS, y la de asimilación a trabajadores por cuenta ajena; nos encontramos con una de las cuestiones más complejas del sistema de Seguridad Social: las situaciones asimiladas al alta. Entre los supuestos previstos legalmente, provocan un especial debate en los procesos de Seguridad Social aquellos casos en los que el presunto beneficiario de invalidez pretende acceder a la pensión permaneciendo como demandante de empleo, una vez agotada la prestación contributiva o asistencial, amparándose, en efecto, en el artículo 36, párrafo 1. ${ }^{\circ}$, del Real Decreto 84/1996 de 26 de enero, pues por circunstancias vitales diversas - que habrán de analizarse detenidamente en el marco de una interpretación no formalista y humanizadora, v.g. sentencias del Tribunal Supremo de 12-7-1988, 13-9-1988 y 26-1-1998, entre otras- puede haber dejado de sellar su cartilla durante un período de tiempo determinado.

A modo de Derecho comparado, en EE.UU. ${ }^{16}$ se define esta modalidad prestacional como «la incapacidad para llevar a cabo cualquier tipo de actividad remunerada, en sus tareas fundamentales, a causa de un impedimento físico o mental demostrable por médicos e informes médicos durante un período ininterrumpido de, al menos, doce meses», si bien su alcance se encuentra condicionado por la Disability evaluation under social security, una lista cerrada de patologías susceptibles de Invalidez, similar a la puerta que deja abierta el artículo 137.3 de la LGSS para el desarrollo reglamentario por el Gobierno, previo informe del Consejo General del INSS, de una similar enumeración legal.

Concluye este artículo 136.1 de la LGSS indicando la necesaria acreditación de una disminución o anulación de la capacidad laboral del sujeto evaluado, lo cual, en realidad, se constituye como marco para la posterior clasificación de los grados de Incapacidad Permanente, en función de la aptitud profesional residual en cada caso. Nosotros nos ocupamos de los grados de Absoluta y Total, únicos acotados en la investigación.

Así, la Incapacidad Permanente Absoluta - IPA - del artículo 137.1.c de la LGSS, definida en el artículo 137.5 de la misma normativa sustantiva, es la situación que inhabilita por completo al trabajador para toda profesión 
u oficio, y a la que se asigna legalmente el $100 \%$ de la base reguladora. De una primera lectura podría parece ${ }^{17}$ que difícilmente se encontrarán supuestos que encajen plenamente con este descriptor, de no ser por las previsiones del artículo 141.2 de la LGSS que permiten la compatibilidad de la pensión con el ejercicio de actividades acordes con el estado del inválido, y que no representen un cambio en su capacidad de trabajo a efectos de revisión. Son además indicativas de que en esta materia no se van a crear precedentes jurisprudenciales aquellas resoluciones judiciales que vienen a indicar que $l a$ misma enfermedad afecta en mayor o menor grado a una persona, mientras que en otra escasamente afecta a su capacidad -Auto del Tribunal Supremo de 9-7-92, recurso 2144/91-, lo cual no impide la existencia de una doctrina de general aplicación sobre este grado de acción protectora de la IPA, como la que diferencia toda posibilidad física para realizar cualquier quehacer laboral, de aquella otra situación de quien, aún con aptitudes para alguna actividad, no la pueda consumar con cierta eficacia — sentencia de la Sala de lo Social del Tribunal Superior de Justicia de Navarra de 15-12-97-, aunque siempre en consideración a las limitaciones que concurren de manera individualizada en el expediente que ha de juzgarse, más que en los padecimientos en sí mismos - sentencia de la Sala de lo Social del Tribunal Superior de Justicia de La Rioja de 2-2-99, entre otras-.

Por su parte, el artículo 137.1.b de la LGSS en relación con el 137.4 delimita la acción protectora de la Incapacidad Permanente Total - IPT-, disponiendo la inhabilidad del trabajador para la realización de todas o de las fundamentales tareas de su profesión habitual, siempre que pueda dedicarse a otra distinta. Su correlato económico se traduce en un 55\% de la base reguladora, o en un $75 \%$ si, entre otros requisitos, el beneficiario cuenta con 55 o más años. Para analizar la profesión habitual a la que el trabajador dedicaba su actividad fundamental previamente al inicio de la incapacidad, obligada es la valoración - siguiendo las Fundamentaciones del Tribunal Supremo, v.g. 21-11-96, Recurso 465/96- de todas las circunstancias de la actividad de trabajo, incluida la incompatibilidad con un ambiente determinado, si bien esta solución puede chocar con la pacífica — ahora sí- doctrina jurisprudencial en el sentido de que no cabe equiparar el concepto jurídico de profesión habitual con el de categoría, y menos aún con el de que trae causa en el puesto de trabajo, pues la pérdida de este último no siempre implicará que el sujeto no pueda seguir desempeñando su profesión en otro centro de trabajo o, incluso, con encuadramiento en otro régimen de la Seguridad Social.

En los casos de FM y/o SFC nos encontramos, además, con una dificultad adicional. Recordemos que el colectivo afectado lo conforman fundamentalmente mujeres, relegadas hasta ahora, en muchos casos, a las funciones de ama de casa y, a pesar de que se han dado ya los primeros pasos para la valoración económica de la producción doméstica, atribuyéndole de lege ferend $a^{18}$, entre otras, la categoría laboral del trabajador autónomo, confor- 
me a lo dispuesto en la disposición adicional primera de la Ley 24/1997, de 15 de julio, de Consolidación y Racionalización del Sistema de Seguridad Social, lo cierto es que en la actualidad no se les atribuye una concreta dimensión jurídico-laboral y, por tanto, no están protegidas por la prestación permanente analizada en su nivel contributivo.

Todo cuanto acaba de indicarse se sustancia por los cauces procedimentales, primero de la vía administrativa —en la que intervienen los Equipos de Valoración de Incapacidades, adscritos a las diferentes Direcciones Provinciales del Instituto Nacional de la Seguridad Social, cuya constitución y competencias aparecen reguladas en el Real Decreto 1300/1995 de 21 de julio y, para su aplicación y desarrollo, en la Orden de 18 de enero de 1996-, $\mathrm{y}$, una vez concluida ésta, - normalmente con resolución denegatoria en los supuestos de FM y/o SFC - se seguirán los trámites de la vía judicial previstos legalmente en el texto refundido de la Ley de Procedimiento Laboral (Real Decreto Legislativo 2/1995 de 7 de abril) y la Ley de Enjuiciamiento Civil con carácter supletorio. Por ello, en lo sucesivo se citarán siempre Sentencias Judiciales —de Juzgados de lo Social (SJS) y de las Salas de lo Social de los Tribunales Superiores de Justicia (STSJ) - correspondientes a los expedientes de las personas que formaron parte de nuestra muestra.

Pero ¿cómo encajar la FM y/o SFC en estos presupuestos jurídicos, si los médicos a los que corresponde, en primera instancia, la valoración del cuadro no se ponen de acuerdo sobre la conveniencia o no de la incapacitación de estas personas? Basta, en este sentido, con comprobar que mientras White ${ }^{19}$ critica la actitud de aquellos sectores clínicos que argumentan que la única razón por la que este síndrome - FM- existe es por contar con un sistema de pensiones un tanto generoso que está preparado para ser «desplumado» por unos individuos que reivindican estar demasiado enfermos para trabajar otros, por el contrario ${ }^{20}$, justifican los efectos contraproducentes de, incluso, la Incapacidad Temporal en estos pacientes, en el convencimiento de que serán más conscientes del dolor como consecuencia de la inactividad, se sentirán aislados, el descenso en su productividad les llevará a una pérdida de la autoestima, tendrán problemas económicos, perdiendo, además, su estabilidad laboral, estarán atemorizados por el momento en el que, en su caso, hayan de reincorporarse al trabajo, sin olvidar, tampoco, los problemas familiares provocados por todo cuanto antecede.

Partiendo de esta variada gama de opiniones, en nuestro trabajo nos planteábamos, a modo de objetivos, de una parte, conocer las, en su caso, especiales características que presentan aquellos afectados de FM y/o SFC que inician - $\mathrm{O}$ se les instruye - un proceso de Incapacidad Permanente, respecto a quienes no lo hacen, por ejemplo en cuanto a comorbilidad, tratamientos... ; y, de otra parte, intentábamos llegar a un consenso entre la clase médica y jurídica sobre la dinámica médico-legal que debía seguirse en lo sucesivo, en consideración a los contenidos de las referencias médico-cien- 
tíficas y las sentencias judiciales, valoradas de forma cualitativa-descriptiva y analítica. No obstante lo anterior, consideramos, también, que se hacía preciso aplicar la estadística, siquiera como instrumento coadyuvante, a los expedientes de Incapacidad Permanente en los que figuraban los diagnósticos de FM y/o SFC, y que se instruyeron por quien suscribe desde el 23 de febrero de 1999 hasta el 31 de julio de 2004 -muestra de 77 expedientes tramitados en distintas provincias españolas-. Solamente desde esta última perspectiva metodológica, estábamos en disposición de aproximarnos a los criterios que pudieron influir en el sentido de la resolución de los casos - estimatoria o desestimatoria—, como son el sexo del juzgador, el contenido de la profesión habitual o los meses consumidos previamente de la prestación de Incapacidad Temporal.

Finalmente, un estudio de estas características precisaba de un cómputo, siquiera aproximado - pues no debe pasarse por alto que contamos con limitaciones metodológicas ${ }^{21}$ - tanto del coste farmacológico medio que provocan los sujetos analizados, como de los montantes económicos que representan los pensionistas que padecen estas realidades clínicas respecto al total de incapacitados permanentes, únicamente en los grados - recordemos- de total y absoluta, para lo que nos apoyamos en los datos facilitados por la entidad gestora Instituto Nacional de la Seguridad Social.

Los resultados pusieron de manifiesto que nos encontramos fundamentalmente con mujeres — como era de prever $(90,90 \%)$ - , con una media de edad de 48,35 años, residentes en un entorno en su mayoría urbano -industrial o sector servicios $(94,80 \%)$ - y que, finalmente, desarrollaban profesiones de contenido eminentemente físico $(59,74 \%)$, aunque son, paradójicamente, las de tipo sedentario y/o intelectual en las que los datos estadísticos nos muestran una cierta tendencia a la estimación, aunque sin contraste - esta particularidad estadística obedecía a la muestra utilizada, un tanto escasa, así como a la categorización que fue diseñada al efecto-.

Esta dicotomía profesional - física frente a sedentaria- y su incidencia en la incapacitación de los afectados de FM y/o SFC se confirman plenamente en las sentencias analizadas, pues, aun cuando para algunos Juzgadores $a$ quo en el dolor fibromiálgico severo y rebelde al tratamiento está contraindicada la realización de esfuerzos, manejo de pesos, posturas prolongadas, también de bipedestación, y movilidad continua de las articulaciones, agravando todos estos parámetros la sintomatología - SJS n. ${ }^{\circ} 1$ de Vizcaya de 22-11-00, SJS n. ${ }^{\circ} 3$ de Vizcaya de 24-5-01, SJS n. ${ }^{\circ} 2$ de Palencia de 15-10-03, SJS n. ${ }^{\circ} 3$ de Guipúzcoa de 13-2-04-, pues es el dolor el que limita la movilidad al margen del estado físico de las articulaciones - SSTSJ del País Vasco de 30-11-01, recurso n. ${ }^{\circ} 2110 / 2001$, SJS n. $^{\circ} 3$ de Vizcaya de 22-10-02, lo cual resulta predicable del SFC, sobre el cual se postula la evitación de actividades de esfuerzo físico (en atención a la fatiga crónica objetivada a través de la febrícula y astenia) - SJS n. ${ }^{\circ} 8$ de Vizcaya de 26-11-01—; para otros, por el contrario, deben prevalecer, no sólo los resultados de las prue- 
bas radiográficas que, en efecto, no objetivan signos que justifiquen el cuadro doloroso y la limitación funcional referida por los pacientes - SJS n. ${ }^{\circ} 3$ de Vizcaya 29-1-01 - , sino que también el estado de la movilidad de las articulaciones - SSTSJ del País Vasco de 14-5-02, recurso n. . 902/2002-. Repárese en que lo que en realidad se discute es cuál es el dato exploratorio que debe considerarse a los efectos de la calificación de la Incapacidad Permanente: limitaciones de movilidad articular o restricciones dolorosas de la movilidad.

Por contra, en los trabajos clínicos se llega a un cierto consenso a la hora de considerar estos factores de sobrecarga mecánica de los músculos ${ }^{22}$ y de realización de esfuerzos propios de las profesiones de contenido eminentemente físico, especialmente repetitivos ${ }^{23}$, como agravantes de las manifestaciones álgicas que, por ende, deben reducirse en el puesto de trabajo; no siendo ésta una idea nueva, desde el momento en que ya en una publicación editada en $1929^{24}$ se trataba la incidencia que en la, entonces, Fibrositis tenía la exposición al esfuerzo, lo cual se ejemplificaba con los mineros que presentaban dolor en la región dorsal y hombros como consecuencia del empleo del pico en posición forzada, y ello sin perjuicio de que se abogaba, también, por promover desde el principio los movimientos y ejercicios activos para evitar la rigidez.

Ahora bien, a pesar de la mayor tendencia a la estimación observada, también encontramos controversia en las sentencias en torno a la contraindicación o no de permanecer en posiciones sedentes constantes, características de las profesiones de tipo administrativo. La solución positiva la encontramos en aquellos fundamentos jurídicos que inciden en que los dolores generalizados, particularmente intensos en columna cervical, lumbar y en extremidades superiores, impiden la adopción de posturas sedentes - SSTSJ de Madrid de 15-10-02, recurso n. . 307/2002-; frente a aquellos otros en los que se sostiene que el dolor aparecería al cabo de un lapso temporal importante en este tipo de posiciones, pues hasta ese momento la capacidad es ordinaria - SJS n. ${ }^{\circ} 8$ de Vizcaya de 23-9-02-.

Por su parte, los promedios estadísticos nos mostraban una permanencia en Incapacidad Temporal previa al procedimiento de Invalidez de 15,05 meses - con tendencia a la estimación a mayor lapso temporal transcurrido-, de lo que deducimos un primer elemento de diferenciación entre nuestra muestra y el colectivo general de pacientes, que acreditaban 9,01 meses consumiendo este tipo de prestación ${ }^{25}$.

En cuanto a los antecedentes personales, llamaba nuestra atención la alta prevalencia de apendicectomías hallada en estas personas -22,07\% - , lo cual confirmaba nuestra hipótesis inicial de una mayor presencia de este tipo de intervenciones quirúrgicas respecto a la población general, como ya se informaba, por otra parte, a inicios del siglo pasado ${ }^{24}$, es decir, un punto sensible a nivel de los músculos oblicuos derechos acompañado de dispepsia vaga podía ser y, a veces, era confundido con la apendicitis, y lo que resulta 
más preocupante, que aquel proceso sería culpable, en algunos casos, de operaciones para la extirpación del apéndice por el cirujano, quien luego habría de apelar a su imaginación para descubrir la concreta lesión hallada.

Continuamos analizando los datos que se extraían de la anamnesis - entrevista clínica médico-paciente_- entre los que destacan como más significativos la historia patobiográfica de acontecimientos vitales adversos -69,76\% - , la disminución o pérdida de la apetencia sexual $-81,81 \%$ - a menudo relacionada con el cansancio, los trastornos psicológicos y la medicación; y, finalmente, las disfunciones cognitivas -en las que se observa una cierta tendencia a la estimación de los supuestos en los que aparecen-descritas por la literatura médica para estos procesos: disfunciones en la atención y concentración, trastornos en la memoria a corto plazo y disfasia nominativa, esta última entendida como la dificultad para adjudicar el fonema correspondiente a la designación de objetos y personas (v.g. cosa, casa...).

Cuestión más compleja es la de la comorbilidad — concurrencia de otras patologías - sobre todo, la psiquiátrica. Así pues, comprobamos que en comparación con otros trabajos que atendían metodológicamente a pacientes con FM y/o SFC en general, nuestros potenciales beneficiarios de Incapacidad Permanente contaban con un mayor número de procesos artrósicos $-74,02 \%$ - herniarios $-45,45 \%$ - y ansioso-depresivos - 66,23\%—. En los dos primeros se observaba, sorprendentemente, una cierta tendencia a la desestimación, mientras que los casos que prosperaban más habitualmente —normalmente en el orden jurisdiccional social, como se indicó más atrás, eran aquellos en que la FM y/o SFC aparecían asociadas a cualquier tipo de psicopatología, o bien en estado puro (en el cuadro clínico solamente figuraban estos síndromes).

Y hablamos de dificultad en lo concerniente a la comorbilidad psiquiátrica, por cuanto que nos adentramos en uno de los mayores debates - en el sector médico y jurídico- sobre estos síndromes: ¿es antes el huevo o la gallina?, es decir, ¿la clínica de la FM y/o SFC provoca las alteraciones psicológicas, o éstas influyeron decisivamente en el diagnóstico?

No debe pasarse por alto, en este sentido, que ya en el siglo XIX Charcot incluyó como una más a la Histeria — que debe su nombre a los Griegos, porque atribuían al útero (Hysterá en griego) la causa de sus trastornosdentro del catálogo de enfermedades, y que los que a día de hoy analizan su influencia en la $\mathrm{FM}^{26}$, sin perder de vista en ningún momento que las algias están presentes, aducen que la Histeria recurre en ocasiones al padecimiento corporal desplazándose allí el centro de su atención y de su sufrimiento, pues el dolor psíquico hace desaparecer la palabra y el pensamiento, transmutándose por fin, en dolor físico; es decir, siguiendo las aportaciones psicosomáticas de Pierre Marty, un complejo proceso a través del cual estos pacientes tratan de resolver a través del cuerpo algo que no pueden manejar de otra forma. 
De cualquier forma, las conclusiones que se alcanzan difieren bastante entre sí. Mientras Rotés ${ }^{27}$ — uno de los padres de los reumatólogos de nuestro país- y Nielson et $\mathrm{a}^{28}$ piensan que los trastornos psicológicos y los funcionales forman parte del círculo de la Fibromialgia, desde el momento en que la depresión desmotiva al enfermo con lo que aumenta su dolor crónico; y, de igual forma, la sensibilización central, al provocarle más dolor, le hunde a nivel psicológico; otros ${ }^{29}$, en cambio, no perciben esta recíproca causalidad con tanta nitidez, pues advierten que la presencia de una psicopatología relevante en un paciente con este diagnóstico no implica que ambas condiciones no pueden coexistir paralelamente sin ninguna influencia entre ellas; dándose, además, la circunstancia ${ }^{30}$ de que la inespecificidad de algunos de los síntomas - sueño no reparador, dificultad en la memoria y en la concentración, mialgias, fatiga...- aparecen también en la depresión, y sugiriéndose ${ }^{23}$, incluso, que la FM - aplicable también al SFCconstituiría una «enfermedad de moda», que enmascararía, bien un trastorno de somatización como consecuencia de la dificultad de afrontamiento de los problemas cotidianos, o bien una forma de vida con ansiedad o depresión.

La clase judicial no es ajena a esta polémica, desde el momento en que elucubra en el sentido de que está aún por determinar por la ciencia médica si la FM si es consecuencia de padecimientos psíquicos, o a la inversa, los padecimientos psíquicos son efecto secundario de la propia referencia álgica -SJS n. ${ }^{\circ} 7$ de Vizcaya de 13-1-03-. Ahora bien, hay quien se posiciona directamente en la primera de las alternativas expuestas - las referencias álgicas referidas por los pacientes están relacionadas con el subjetivismo y sus problemas psíquicos, SJS n. ${ }^{\circ} 5$ Vizcaya de 15-10-01 y 30-11-01, respectivamente-, aludiéndose, incluso, en algunos supuestos, a que el diagnóstico de neurosis histérica influiría sobre la percepción del dolor -SJS n. 7 de Vizcaya de 18-2-03, SSTSJ del País Vasco de 5-3-04, recurso n. ${ }^{\circ} 3005 / 2003$-.

De todas formas, observamos una favorable acogida de las pretensiones de la prestación analizada - no en todos los casos, conforme a los datos estadísticos-, cuando concurre en comorbilidad el psicodiagnóstico de trastorno depresivo mayor, lo que evidencia la existencia de un menoscabo psiquiátrico severo y un superior deterioro de las facultades cognoscitivas -SJS n. ${ }^{\circ}$ 9 de Vizcaya de 28-2-02, SJS n. ${ }^{\circ} 4$ de Vizcaya de 25-9-02-. Se llega a asociar con esta consideración nosológica psíquica la clínica de severa pérdida de memoria, atención y concentración, propia de la FM y/o SFC, según hemos explicado anteriormente, lo cual, nos parece, en principio, un contrasentido - SJS n. ${ }^{\circ} 7$ de Vizcaya de 4-2-04-.

Tampoco parece que estén en lo cierto aquellos magistrados que, en aplicación de una doctrina uniforme, señalan que en cuanto a las más numerosas sentencias que aprecian situación de Invalidez lo hacen, por lo general, en supuestos en que la FM aparece en calidad de enfermedad concomitante 
o asociada a otras patologías, normalmente de índole depresiva - $\mathrm{SJS}$ n. ${ }^{\circ} 2$ de Vigo de 9-7-03 y SJS n. ${ }^{\circ} 3$ de Guipúzcoa de 26-11-03-, pues la estadística revela, también, una mayor tendencia estimatoria cuando nos encontramos ante una FM y/o SFC puros.

Nos ocupamos ahora de la exploración física, a través de la cual se determinó en nuestra serie una media de 16,23 puntos sensibles de los reproducidos en la Tabla número 1 que, nuevamente, llama nuestra atención, y que provocan una cierta tendencia a la desestimación de la pensión cuanto mayor es su número; lo cual pondría, en definitiva, de manifiesto la controversia al menos en el ámbito judicial — de su utilidad clasificatoria; y ello a pesar de lo que se aduce en contrario en algunas resoluciones judiciales: la jurisprudencia y la doctrina médica son unánimes a la hora de determinar que la FM adquiere la categoría de enfermedad grave e invalidante cuando supera los 18 puntos dolorosos - SJS n. ${ }^{\circ} 2$ de Vigo de 9-7-03, SSTSJ de Cantabria de 24-6-04, recurso n. ${ }^{\circ}$ 112/04-.

Constituyen, también, datos exploratorios relevantes las restricciones dolorosas de la movilidad $-82,85 \%$ - las contracturas musculares $-70,76 \%$ - o la disminución de la fuerza segmentaria $-63,63 \%$ -

Su importancia a los efectos prestacionales que nos ocupan, se confirma desde el momento en que, ciertamente, no $\operatorname{contamos}^{8}$ con ningún signo o prueba de laboratorio que coadyuve en el diagnóstico, lo que hace que la FM - y por extrapolación, el SFC - entren a formar parte de esa categoría de los síndromes funcionales-somáticos que se estructuran más por los síntomas que por las pruebas complementarias. No es - asegura Rivera ${ }^{32}$ - como una gripe en la que los signos son tan precisos que sólo existen dos posibilidades, la tienes o no la tienes.

Por ello, los magistrados echan de menos una valoración pormenorizada de los efectos funcionales de estos síndromes. La proyección incapacitante de dicho padecimiento, se indica, requiere una particular rigurosidad en la prueba - SJS n. ${ }^{\circ} 5$ de Zaragoza de 14-3-02, SSTSJ de Cataluña de 17-1-03, recurso n..$^{\circ}$ 2672/2002, entre otras-.

Nuestra muestra, por otra parte, se encontraba en su mayoría polimedicada $-74,02 \%$ - dato este que, en comparación con los resultados que se deducen de la investigación liderada por Wolfe ${ }^{33}$, sugiere un nuevo elemento diferenciador entre nuestra serie que postula la prestación de Incapacidad Permanente, y el grupo general de pacientes de FM y/o SFC en el que no concurría esta multifarmacología. Además, el coste farmacéutico que representaban las personas a las que se les habían pautado amitriptilina, paracetamol, antiinflamatorios no esteroideos, tramadol y opioides ascendía a $1.634,19$ euros por persona y año.

Finalmente nos encontramos con una estimación del 44,15\% de los expedientes que se instruyeron durante el período de referencia, de los cuales el 73,52\% correspondían al grado de Incapacidad Permanente Total y el 26,47\% al de Absoluta, dato curioso en relación con la estadística general de 
pensionistas, en la que no se observaba una diferencia de esta magnitud y que interpretamos, de acuerdo con la polémica expuesta sobre estos procesos patológicos, en el sentido de que se percibe una cierta cautela de los órganos judiciales a la hora de la calificación de estos supuestos y, por ende, en los casos en que se reconoce la pensión, se hace, por lo general, en el marco del menor grado de acción protectora que representa la Incapacidad Permanente Total.

Los datos estadísticos, en esta ocasión, nos muestran una mayor tendencia a la estimación cuando la sentencia es dictada por una magistrada de lo Social, quizá en atención a un rol de protección por razón de género, teniendo en cuenta la mayoría aplastante de mujeres como «sujetos pasivos» de las entidades clínicas que nos ocupan; si bien, cuando se formalizaba recurso de suplicación ante las salas de lo Social de los Tribunales Superiores de Justicia, se observaba una cierta tendencia a la revocación cuando concurría una FM y/o SFC puros frente a si se acreditaba comorbilidad con otras patologías orgánicas o psiquiátricas, o, incluso, cuando el proceso patológico determinante del fallo fue la psicopatología -a lo sumo revocaciones parciales-.

$\mathrm{Si}$ a todo cuanto hemos expuesto se añade la alta epidemiología de Incapacidad Permanente Absoluta y Total por FM y/o SFC — una vez extrapolamos nuestros datos a las estadísticas generales de pensionistas españoles-, es decir, estas personas percibirían un $14,66 \%$ de todas las pensiones de este tipo que abona el INSS, con su correlato económico del 22,30\% de las partidas que destina esta entidad gestora para estos grados prestacionales, llegamos a la conclusión de que era necesario plantear un trabajo de estas características.

Hemos apostado por una nueva forma de hacer investigación, cuyos rigores metodológicos, muy probablemente, no alcancen a los ya existentes, aunque lo cierto es que una vez finalizado este trabajo multisdisciplinar, estamos en disposición de afirmar que existen dos disciplinas que no están tan distanciadas como podría parecer a primera vista: la jurídica y la médica. 


\section{Tabla número 1 \\ Criterios clasificatorios de la Fibromialgia ${ }^{3}$}

\section{Para proceder a la clasificación, es preciso que concurran en el paciente de forma simultánea, los dos siguientes criterios (ACR):}

1. Historia de dolor generalizado durante al menos tres meses:

- En el lado izquierdo del cuerpo.

- En el lado derecho del cuerpo.

- Encima de la cintura.

- Debajo de la cintura.

- Dolor de esqueleto axial (columna cervical, cara anterior del tórax, columna dorsal o lumbar).

2. Dolor en al menos 11 de los 18 puntos sensibles a la palpación digital (fuerza de $4 \mathrm{~kg}$ ). Puntos positivos son los que el paciente presenta como dolorosos a la palpación, pues una molestia no se considera como dolor. Las principales localizaciones son las siguientes:

- Occipucio bilateral, en inserción de los músculos suboccipitales.

- Cervical bajo: bilateral, en la cara anterior del espacio intertransverso de C5-C7.

- Trapecio: bilateral, en el punto medio de su borde superior.

- Supraespinoso: bilateral, en su origen sobre la espina de la escápula cercana a su borde medial.

- Segunda costilla: bilateral, a nivel de la $2 .^{a}$ unión costocondral, lateral a la articulación de ambas superficies.

— Epicóndilo lateral: bilateral, a $2 \mathrm{~cm}$ distal del epicóndilo.

- Glúteo: bilateral, en el cuadrante supero-externo de las nalgas en el pliegue anterior del músculo.

- Trocánter mayor: bilateral, posterior a la prominencia del trocánter.

- Rodilla: bilateral, en el paquete de grasa medial proximal a la interlínea articular. 


\section{Tabla número 2}

\section{Criterios diagnósticos del Síndrome de la Fatiga Crónica9}

1. Fatiga crónica persistente (seis meses mínimo), o intermitente, inexplicada, que se presenta de nuevo con inicio definido y que no es resultado de esfuerzos recientes; no mejora claramente con el descanso; ocasiona una reducción considerable de los niveles previos de actividad cotidiana del paciente.

2. Exclusión de otras enfermedades potencialmente causantes de fatiga crónica.

De forma concurrente, deben estar presentes cuatro o más signos o síntomas de los que se relacionan, todos ellos persistentes durante seis meses o más y posteriores a la presentación de la fatiga:

1. Trastornos de concentración o memoria recientes.

2. Odinofagia.

3. Adenopatías cervicales o axilares dolorosas.

4. Mialgias.

5. Poliartralgias sin signos inflamatorios.

6. Cefalea de inicio reciente o de características diferentes de la habitual.

7. Sueño no reparador.

8. Malestar postesfuerzo de duración superior a veinticuatro h. 


\section{Referencias bibliográficas*}

1. El paciente en España. Madrid: Fundación Farmaindustria; 2004. p. 557.

2. McKinley W, Cram J, Margoles M et al. Innovations in Soft-Tissue Jurisprudence. Trial Diplomacy Journal 1992; 15: 199-208.

3. Wolfe F, Smythe HA, Yunus MB et al. The American College of Rheumatology 1990 Criteria for the Classification of Fibromyalgia. Report of the Multicenter Criteria Commitee. Athritis and Rheumatism 1990; 33 (2): 160-172.

4. Carceller JM, Fidalgo MI. Tratamiento de la Fibromialgia desde la clínica del dolor. Dolor 1998; 13: 116-122.

5. Rodríguez R, Lindman R, Mulero J et al. Fibromialgia. Actualización y nuevas perspectivas. Madrid: ICN Huber; 1994.

6. Mulero J, Andreu JL, Sanz J. Fisiopatología de la Fibromialgia. Dolor 1998; 13: 110-115.

7. Cohen H, Neumann L, Shore $\mathrm{M}$ el al. Autonomic Dysfunction in Patients With Fibromyalgia: Application of Power Spectral Analysis of Heart Rate Variability. Seminars in Arthritis and Rheumatism 2000; 29 (4): 217-227.

8. Prevalencia e impacto de las enfermedades reumáticas en la población adulta española. Estudio EPISER 2000. Madrid: SER, MSD, Ministerio de Sanidad y Consumo; 2001. pp. 77-91.

9. Fukuda K, Strauss SE, Hickie I. et al. The Chronic Fatigue Syndrome: A Comprehensive Approach to Its Definition and Study. Ann Intern Med 1994; 121: 953-959.

10. Wessely S, Chalder T, Hirsch S et al. The Prevalence and Morbidity of Chronic Fatigue and Chronic Fatigue Syndrome: A Prospective Primary Care Study. American Journal of Public Health 1997; 87 (9): 1449-1455.

11. Manrique F. Manual de Derecho de la Seguridad Social. Bilbao: Universidad de Deusto, Departamento de Publicaciones; 1984.

12. Soriano E, Gelado MJ, Girona MR. Fibromialgia: un diagnóstico cenicienta. Atención primaria 2000; 26 (6): 415-418.

13. Belmonte MA. Dolor musculoesquelético generalizado no articular, Fibromialgia y síndromes afines. Jano 1990; 904: 88-94.

14. García JM. Mecánica procesal de las Incapacidades Laborales. Valencia: Ediciones Tro; 1999.

15. Álvarez M, De Val AL. Manual de Derecho de la Protección Social. Murcia: Ediciones Laborum; 2003.

* En este trabajo se ha seguido el sistema de citación bibliográfica de Vancouver. 
16. Starlanyl DJ, Copeland ME. Fibromyalgia and chronic myofascial pain syndrome. Vermont: New Harbinger Publications, Inc.; 1995. pp. 333344.

17. Blanco JM, Dueñas LJ, López M et al. Las Incapacidades Laborales. Un punto de vista práctico. Valladolid: Lex Nova; 2002.

18. Alonso E, Serrano M, Tomás G. El trabajo del ama/amo de casa. Un estudio jurídico y su consideración ética. Vitoria-Gasteiz: Diputación Foral de Vizcaya, Gabinete del Diputado General; 2003. pp. 15-149.

19. White KP. Fibromyalgia: The Answer Is Blowin' in the Wind. The Journal of Rheumatology 2004; 31 (4): 636-639.

20. Bernier R. Fibromyalgia. Collége des Médecins du Québec 1996.

21. Galindo C. Fibromialgia. Dolor 2004; 19: 171-187.

22. Alonso JL, Álvarez B, Alegre J. Fibromialgia. Seminarios de la Fundación Española de Reumatología 2000; 1-4: 199-211.

23. Zubieta AJ, Aragón A, Cantalejo M. La Fibromialgia: una enfermedad del pasado, actual y con futuro. Rheuma 2003; 6: 9-22.

24. Thomson FG, Gordon RG. Las enfermedades reumáticas crónicas, diagnóstico y tratamiento. Barcelona: Manuel Marin - editor; 1929. pp.18-28.

25. Albornoz J, Povedano J, Quijada J et al. Características clínicas y sociolaborales de la Fibromialgia en España: Descripción de 193 pacientes. Rev Esp Reumatol 1997; 24: 38-44.

26. Muro J. Fibromialgia: Un misterio doloroso. Reflexiones en clave «F» 2003; 1: 14-18.

27. Rotés-Querol J. La Fibromialgia en el año 2002. Rev Esp Reumatol 2003; 30 (4): 145-149.

28. Nielson WR, Harth M. Fibromyalgia: Beyond the Rhetoric. The Journal of Rheumatology 2004; 31 (4): 631.

29. Yunus MB. Psychological aspects of Fibromyalgia syndrome: a component of the dysfunctional spectrum syndrome. Baillière's Clinical Rheumatology 1994; 8 (4): 811-837.

30. Komaroff AL. The Biology of Chronic Fatigue Syndrome. The American Journal of Medicine 2000; 108: 169-171.

31. Leza JC. Fibromialgia: Un reto también para la neurociencia. Rev Neurol 2003; 36 (12): 1165-1175.

32. Rivera J. Controversias en el diagnóstico de Fibromialgia. Rev Esp Reumatol 2004; 31 (9): 501-506.

33. Wolfe F, Anderson J, Harkness D et al. A prospective, longitudinal, multicenter study of service utilization and costs in Fibromyalgia. Arthritis \& Rheumatism 1997; 40 (9): 1560-1570. 